\title{
Conceptualising Beauty in Consumer Research: A Framework and Research Agenda: An Abstract
}

\author{
Marina Leban and Benjamin Voyer
}

\begin{abstract}
The cosmetic industry has been growing rapidly in the last few decades. Currently, the industry itself generates about $\$ 230$ billion each year (Forbes 2006). Multinational market leaders in cosmetics such as L'Oreal and Estee Lauder own practically most of the cosmetic brands. Recent studies have shown that $90 \%$ of female consumers use cosmetic products daily (Guthrie and Kim 2009).

With L'Oreal's recent business plan to expand the distribution of cosmetic products in emerging markets (Forbes 2014), research on consumer trends in cosmetic products and, in general, on beauty is ever so salient. Yet consumer research has neglected to look at beauty product consumption more closely. Researchers have not been able to explain the underlining mechanisms of motivation as to why consumers use beauty products on an everyday basis.

This paper will review beauty in terms of evolutionary, cognitive and cultural influences, as well as the importance of beauty packaging to better understand the concept of beauty and to pinpoint possible gaps existing in the literature. The aim of this paper is to understand beauty consumption as a whole by merging evolution, cognition and culture in a framework so as to encourage marketing research to take into consideration all three levels of beauty perception in future studies.
\end{abstract}

\footnotetext{
M. Leban (ه)

ESCP Europe Business School, Paris, France

e-mail: marina.leban@edu.escpeurope.eu

B. Voyer

ESCP Europe Business School, London, UK

e-mail: bvoyer@escpeurope.eu
} 